\title{
Entwined Influences of Users' Behaviour and QoS: A Multi-model Approach
}

\author{
Julien Siebert, Vincent Chevrier, and Laurent Ciarletta \\ LORIA: MADYNES \& MAIA teams - Campus Scientifique - BP 239 - 54506 \\ Vandœuvre-lès-Nancy Cedex \\ \{julien.siebert, vincent. chevrier, laurent.ciarletta\}@loria.fr
}

\begin{abstract}
In distributed, dynamic networks and applications, such as Peer-to-Peer (P2P), users' behaviour and quality of service/quality of experiment 1 are known to influence each other. In worst cases, these mutual influences could lead the system to crash. We propose a novel approach to model relationships between users and QoS. It is based upon multiagent systems in order to study the impact of situated behaviours on the global network and to integrate different levels of representation (users' behaviour, overlay protocols, network topology). This paper describes our approach to represent the different models required in such systems and a first implementation in an existing overlay simulator with the first results of experimentations.
\end{abstract}

\section{Motivation}

For the last couple of years, distributed applications (such as P2P file sharing) have rapidly expanded. In these applications [12], users' behaviour is known to have a great impact on the quality of service (QoS), as for instance the problems of free-riding and poisoning (studied in section 3). QoS also impacts the users' behaviour. For example, a user can stop or defer the use of an application because of a large bandwidth consumption. Moreover, applications are expected to become ubiquitous and to be able to guide user's behaviour.

Understanding these mutual influences can help to foresee the behaviours which can be dangerous/beneficial for the network performance (NP) and the impact of NP on these behaviours. This work can also be useful to quantify the dysfunctions based on the frequency/ratio of these behaviours, or to test future applications.

To do this, we argue that we need to take into account different levels of representation. Indeed, the users' behaviour depends on the objective QoS and on the local user's environment (moment, location, stress,...). For the QoS (see 3]), it depends mostly on NP and on the media environment. In short, we claim it is needed to study relations between models of (1) user, (2) application and (3) environment in order to assess the mutual influences (fig. 1).

\footnotetext{
${ }^{1}$ In this article, we use the term QoS to design both quality of service and quality of experiment.
} 


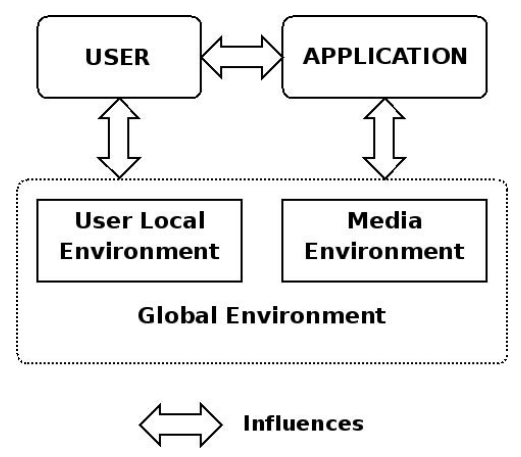

Fig. 1. Relations between the different levels of representation

Models and simulators, in the network domain (such as NS, etc. see review 4), are well fitted to study QoS and network performances. The users' behaviour, if present, is just an input for these models. It is often reduced to a quantity of traffic generated. As a consequence, it seems difficult to model dynamic and heterogeneous users' behaviour, and to take into account the mutual influences of QoS and behaviour with such a limited approach.

Obviously, models and simulators in the field of cognitive sciences (economy, social sciences, game theory,...) are well suited to represent user's behaviours, goals, actions and interactions [5]6. However, modelling realistic network's parameters is not the primary objective of this field. As a consequence, parameters such as delays, bandwidth or overlay protocols are seldom if ever represented.

After looking at each domain, we conclude that a single point of view does not seem sufficient. Since models already exist for each level, we need to integrate and make them interact.

\section{Proposition of Study}

We propose to use the multi-agent (MA) paradigm 7 to describe $\mathrm{P} 2 \mathrm{P}$ systems and to make interact our different levels of abstraction. MA is appropriate to simulate complex distributed systems such as, for example, societies [8. It could be defined as a set of organised autonomous entities (the agents) interacting with and within an environment to achieve some goals. In our case, we define users as agents2, protocols as interactions and underlying networks as the (media) environment (see fig. 2).

Our goal is to implement, to test our multi-agent approach and to compare it with existing models and real data. Then, we want to build a generic framework enabling us to assess the mutual influences of users' behaviour and QoS, not only in $\mathrm{P} 2 \mathrm{P}$ but also in other distributed, dynamic networks and applications (adhoc, mesh). We wish to test different heterogeneous behaviours and/or different overlay protocols and/or different network topologies. This last point highlights

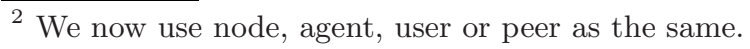




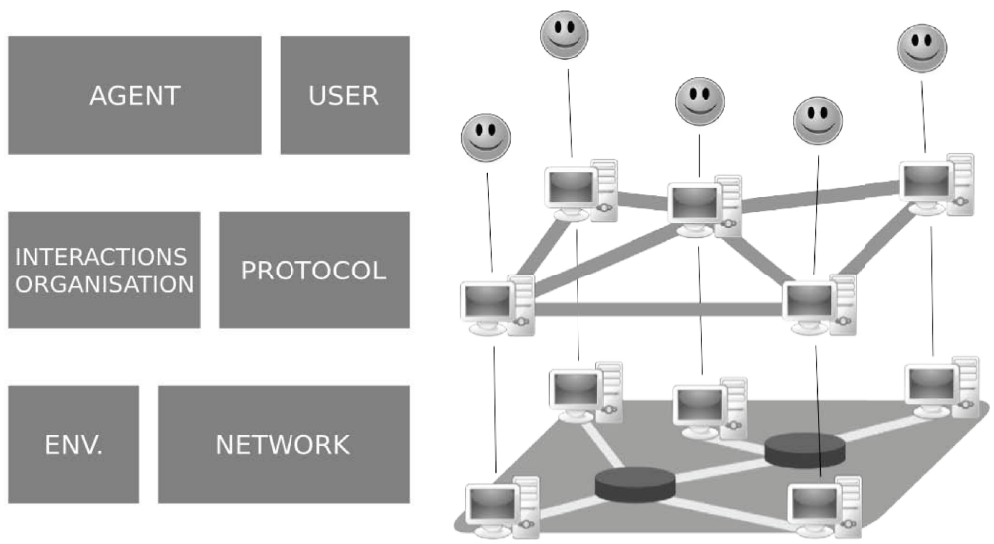

Fig. 2. Multi-agent for P2P 9 ]

multi-model issues: how several models at different space and time scales can interact?

\section{A First Case Study}

As a first case study of our approach, we restrict the domain to P2P file sharing applications (see 9]). We are studying the problems of free-riding: people that do not share their resources; and poisoning: content that differs from the original copy (also called pollution). We would like to assess the impact of sharing and poisoning awareness on the content spreading. These problems are known to be quite hard to solve in decentralised systems and methods to avoid free-riding and poisoning are still an open area (see [1062]).

MA approach enables us to understand the impact of local behaviours on the global state of the system. However, we were firstly interested in its feasibility: we build a simulator and make experiments to answer the questions of realism and scalability of our implementation.

Due to time constraint 3 , we chose to adapt an existing overlay simulator: PeerFactSim.KOM 4 (PFS). This tool had several advantages for the study of our problematic. The first one is the scalability (up to $10^{5}$ nodes) and the second one is its architecture that separates each level of abstraction and facilitates the implementation of a user model.

We adapted PFS to fit with our approach by explicitly modelling data and their concrete exchanges (each node has now a list of files which can be shared over the network), by modelling pollution within these files and adding new metrics such as, for example, number of polluted files per node.

\footnotetext{
3 This work was done during my Master Thesis.

${ }^{4}$ http://www.peerfact.org
} 


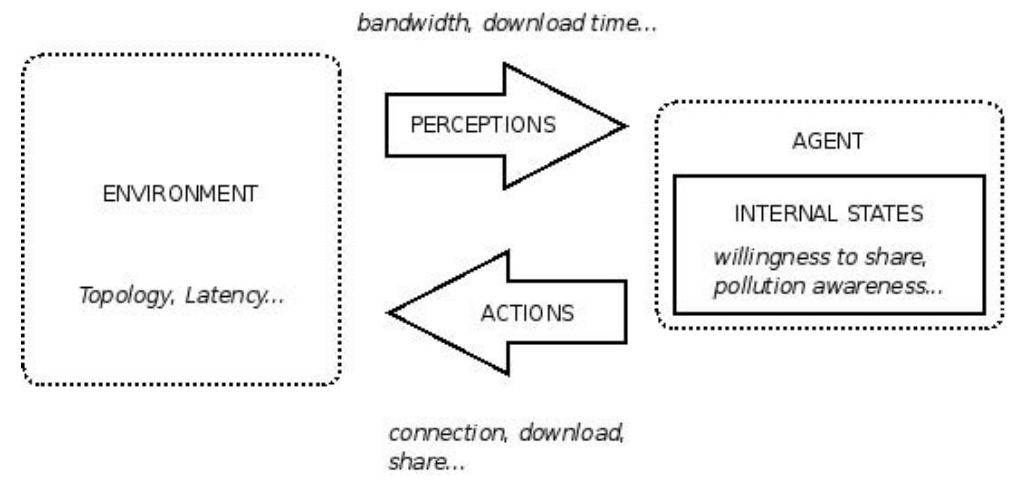

Fig. 3. Perception Decision Action cycle in our model 9]

The user, in our model has a relatively simple behaviour with few internal states and a compact representation of their external environment and neighbours. It decides which action to undertake considering its local perception and its internal states (see fig [3).

We establish a first experiment's scenario in order to test our approach: we mainly look at technical feasibility (debug), realism and scalability. We take an initial network of $N$ nodes in which $P$ peers publish each $f$ resources. The scenario is composed of five steps: one agent looks for a file; after a while the agent asks one of the available sources for a download; then it checks the download process; if it gets the file, it can check the pollution and decides to share, otherwise it can decide to restart the whole process.

On scalability: we perform experiments over kademlia networks from $N=25$ to 50000 nodes (Java 6, 2GbRAM, core duo 2.4GHz). Simulating 50000 nodes with our model of user nearly tooks 15 minutes.

On realism: we studied the impact of the number of initial publishers $P$ in an ideal case (no pollution, no selfish users). The basic idea is that, when $P$ increases, the load per node spreads equally over the networks (ideal P2P behaviour). We measure the number of received messages per nodes as the load carry by each peer. We saw that effectively, in our tool, the more people publish (a greater $P$ ), the more equally the load spreads. This means that the modified tool behave correctly.

\section{Conclusion and Future Work}

We have presented the basis of a multi-model approach to study the entwined influences of user's behaviour and QoS within the field of distributed, dynamic, ubiquitous network and applications. We integrate and make interact an agentbased model with an application and a network one. We implemented this proposal on a kademlia network to build a proof of concept. 
Results obtained show that approach enables to study the impact of situated users' behaviours on the global P2P network. We aim now at continuing experiments over $\mathrm{P} 2 \mathrm{P}$ file sharing applications. We have recently undertaken experiments to study the impact of poisoning peers: we look at the number of peers that poison the network and how they do it (e.g. number of decoy files they inject). The goal here is to show the conceptual benefits of our approach. We also aim at comparing our results to existing models and real data (in order to validate our model of user).

Currently, underlying network topology and user model are quite simple. However, our long term goal is to build a generic framework to implement and test different models of users and/or different overlay protocols and/or different network topologies. The scientific questions behind is to make interact several models at different time and space scales.

\section{References}

1. Hughes, D., Coulson, G., Walkerdine, J.: Free riding on gnutella revisited: The bell tolls? IEEE Distributed Systems Online 6(6), 1 (2005)

2. Lee, U., Choiz, M., Choy, J., Sanadidiy, M.Y., Gerla, M.: Understanding pollution dynamics in p2p file sharing. In: 5th International Workshop on Peer-to-Peer Systems (IPTPS 2006), Santa Babara, CA, USA (2006)

3. Teletraffic engineering handbook. Technical report, ITU-D Study Group 2 Question $16 / 2(2006)$

4. Naicken, S., Livingston, B., Basu, A., Rodhetbhai, S., Wakeman, I., Chalmers, D.: The State of Peer-to-Peer Simulators and Simulations. Accepted for editorial publication in ACM SIGCOMM journal for Computer Communication Review (2007)

5. Hales, D.: From Selfish Nodes to Cooperative Networks - Emergent Link-Based Incentives in Peer-to-Peer Networks. In: P2P 2004: Proceedings of the Fourth International Conference on Peer-to-Peer Computing (P2P 2004), Washington, DC, USA, pp. 151-158. IEEE Computer Society, Los Alamitos (2004)

6. Feldman, M., Papadimitriou, C., Chuang, J., Stoica, I.: Free-riding and whitewashing in peer-to-peer systems. In: PINS 2004: Proceedings of the ACM SIGCOMM workshop on Practice and theory of incentives in networked systems, pp. 228-236. ACM Press, New York (2004)

7. Ferber, J.: Multi-Agent Systems. In: An Introduction to Distributed Artificial Intelligence. Addison Wesley, Reading (1999)

8. Phan, D., Amblard, F.: Agent-Based Modelling and Simulation in the Social and Human Sciences. Bardwell Press, Oxford (2007)

9. Siebert, J., Chevrier, V., Ciarletta, L.: Modélisation multimodèle des réseaux dynamiques: cas des réseaux pair-à-pair (in french). In: JDIR 2008 - 9èmes Journées Doctorales en Informatique et Réseaux, Villeneuve d'Ascq, France (2008)

10. Thommes, R., Coates, M.: Epidemiological Modelling of Peer-to-Peer Viruses and Pollution. In: IEEE Infocom, Barcelona, Spain (2006) 